\title{
Fundholding and cash limits in primary care: blight or blessing?
}

\author{
Martin Roland
}

This is the fifth in a series of articles commissioned in response to the General Medical Services Committee's strategy paper "Building Your Own Future"

University of Cambridge School of Clinical

Medicine, Addenbrooke's Hospital, Cambridge CB2 2QQ

Martin Roland, DM, director

of studies in general practice

BM于 1991;303:171-2
When I started asking doctors for their views on fundholding and cash limits it soon became clear that there is little agreement about the effect that these two changes will have on the NHS, or even whether they are related. Indeed the views seemed so polarised that I have decided to present two contrasting points of view, followed by my own synthesis about what the changes will mean to the NHS.

\section{Promise of nirvana}

If you want to know where the power lies look at who is signing the cheques to pay for hospital services. And power to improve quality of care is what it's all about. Fundholders have been taken aback by the power that they wield even as single practices. And the power of the large consortiums is such that it can threaten the existence of a provider. What has the power been used for? It has been used to improve patient care. All those things that have angered general practitioners and their patients for years are suddenly out in the open and up for negotiation: Why should patients wait a month to hear when their appointment is? Why should they wait another nine months to see the specialist? Why do patients always wait two hours in the clinic? Why are patients so rarely involved in decisions about their management? Why doesn't the hospital give two weeks' supply of drugs on discharge? Why don't discharge summaries arrive within a week? It is because of fundholding that these things are finally being tackled. Fundholders' agendas have concentrated firmly on quality issues, and discussion of the details of contracts has opened up a completely new dialogue between primary and secondary care.

Patients are already receiving the benefits. In some areas direct access to radiology, endoscopy, and surgical appliances, which has been impossible for years, has become a reality overnight. And the benefits aren't just for fundholders' patients. Fundholders in Leeds negotiated direct access to physiotherapy at Leeds General Infirmary, and this has now become available to all Leeds general practitioners. When district health authorities see what fundholders can achieve they won't be far behind.

Cost is secondary to quality in negotiations, but it is often possible to achieve both. When a gynaecological operation costs less in the private wing than in the NHS wing of Guy's Hospital, why shouldn't the patient have the benefit of her own television? And when 90 minute psychiatric consultations cost $£ 85$ at the private Priory Hospital, with follow ups costing $£ 45$, is it any surprise that fundholders are moving away from the nearby Queen Mary's Hospital, Roehampton, where new or old appointments cost $£ 68$ ?

Cash limits on staff costs, development of services, and premises have nothing to do with fundholding. But here again are some real opportunities for general practice if only the profession could get its act together. These limited budgets are now set by regional health authorities, and there is real opportunity for general practitioners to get together with family health services authorities to make a case for more money. Take Cambridgeshire as an example: in the four years from 1988-9 to 1991-2 the budgets for staff and premises have been $£ 3.9 \mathrm{~m}, £ 5.1 \mathrm{~m}, £ 6.7 \mathrm{~m}$, and $£ 9.8 \mathrm{~m}$. This increase reflects the regional health authority's confidence in primary care. Of course the money will have to be argued for, but practices able to show health needs or prepared to justify their expenditure in terms of improved health outcome will be able to improve their services to patients.

\section{The doomsday scenario}

Well how naive can you get? Of course the fundholders are gung ho about their contracts-flexing their new found muscles and bossing the hospitals around. But what do they think will happen at the end of the year? Political imperatives will prevent the money running out this year, but it will certainly run out next year. Fundholding has everything to do with cash limits. You only have to read what Andrew Foster, deputy chief executive of the NHS, wrote recently: "FHSAs have a key role in regulating the volume and type of demands made on an expensive secondary care resource."' The government has effectively put a cash limit on prescribing and the fundholding scheme is clearly designed to see if it is possible to do the same with referrals. Who will know about the patients who don't get referred? We are told that the family health services authorities "will be monitoring improvements in the quality of care for fundholding practices' patients,"' but you can be sure that they won't be looking too hard at the patients who don't get referred. It will be all right for patients with diabetic retinopathy - they are easy to monitor-but the average fundholder will think twice about referring a clumsy child to a child development centre knowing that the practice may have to pay for ongoing physiotherapy, speech therapy, and occupational therapy.

In the meantime fundholders will cause chaos for the rest of us. If fundholders have negotiated minimum waiting times for appointments and for operations that means an inferior service for patients of nonfundholders. If directly managed hospitals are uneasy about running a two tier system NHS trusts will have no such qualms. Furthermore, planning will go completely out of the window. For example, Triangle Health Care, a consortium of 32 fundholding general practitioners, has $25 \%$ of the local population under its care. For services that don't meet its standards it has given only six month contracts to the local hospitals, with the threat of taking its business elsewhere after that time. How can a district general hospital plan on that sort of time scale? Naturally, some private hospitals have been able to undercut the NHS. There will be an increasing number of private hospitals specialising in low cost, low risk, procedures. We will see the "Harrow Hospital for Hernia," and the "Sydenham Cystoscopy Centre"-just the sort of fragmentation that the NHS was set up to reduce.

As far as cash limits on staff and premises are concerned, funding of these was the element of the 1964 general practitioner contract which probably did more than anything else to improve the standard of general practice. There has been no evidence of abuse of either, and at least general practitioners could plan for staff up to two per doctor. Now they don't know 


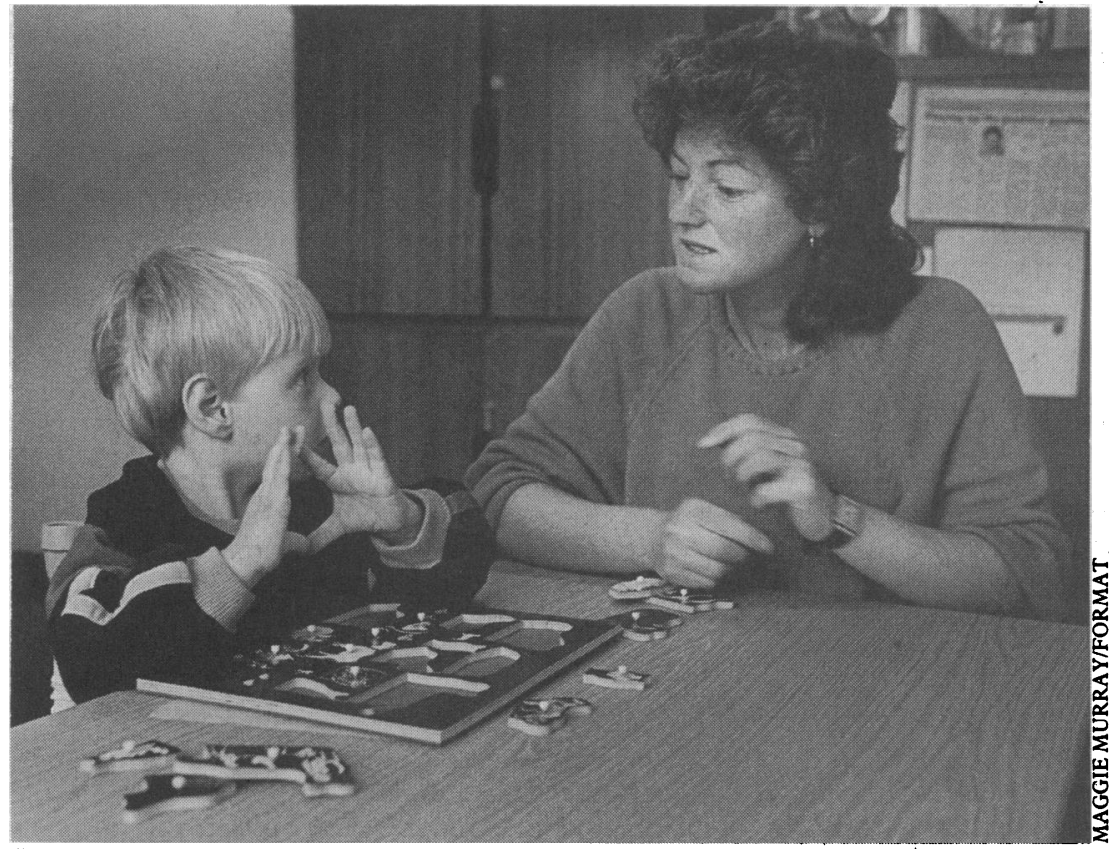

Fundholders may not refer a child to a development centre if they have to pay for ongoing treatment

where they are. And all too often the family health services authority can't plan either because it hasn't been told its cash allocation. And for services that are likely to exceed budgets, such as extracontractual referral and requests for new staff, health authorities have deployed their ultimate weapon-the bottomless in-tray.

\section{The real truth?}

Fundholding is the most radical of the NHS reforms, and fundholders are driving the internal market because they are less constrained than district health authorities to keep to existing referral patterns. Most fundholders have addressed quality of care as the main issue in setting contracts, and this will inevitably lead to a two tier standard of care. Indeed, it must lead to a two tier system if it is to work as is intended - that is, as a lever to produce change across the whole NHS. The question is how district health authorities will respond. If they are sufficiently sensitive to the needs of general practitioners and their patients, they won't be far behind in specifying levels of quality of care comparable with those achieved by the fundholders. So far, districts have not shown this sensitivity, and it is up to general practitioners to ensure that their districts attract hospitals' attention to what their patients need. Fundholders and district health authorities need not be in competition, and some fundholders have been working closely with district purchasing teams in negotiating contracts with hospitals.

Many boundaries that seemed immutable have changed, not least in terms of where care is given, with improved access to some hospital services, increased use of private hospitals, practices undertaking more investigations, and consultants visiting practices or even being employed by fundholding groups. These changes will continue, and a series of new models for providing secondary care may develop. Again there is no reason why changes of this type should not be negotiated on behalf of non-fundholding general practitioners by district health authorities, though fundholders are likely to take the lead.

Fundholders have entered into a fundamentally new relationship with consultants, in many cases to the acute unease of the specialists. Nevertheless, general practitioners need to cooperate with consultants to decide what should be in contracts. There is likely to be increased accountability on both sides in this developing relationship, and these changes will affect both fundholders and non-fundholders. Hospitals will become more accountable for the standard of care they provide, and general practitioners, especially fundholders, will become more accountable for their use of hospital services.

The crucial test of fundholding relates to the fixed budget. A particular danger is that a constrained budget will prevent general practitioners from referring patients and that underreferral will go unnoticed because it is hard to detect. The government has developed a new tool to limit the costs of secondary care, and it remains to be seen how they will use it.

Limiting of funds for staff, premises, and development of services is a less radical change. Instead of having these resources by right, general practitioners will now have to make a case for them against locally specified priorities. General practitioners will need to develop the skills to enter into this sort of discussion, and they will lose out if they fail to learn the new language of managers of family health services authorities. The authorities have got, and will exercise, managerial control over these budgets in deciding, for example, whether to allocate money for premises or money for staff, whether to target one geographic area for more resources, whether to give priority to employing particular types of staff, or whether to support the least effective practices or to reinforce innovative practices that have achieved the highest standards of care. The views of the profession will simply be sidestepped if general practitioners do not contribute constructively to these debates.

And the real truth? The real truth is that rapid changes are occurring in the NHS, and those doctors, fundholders or not, who are able to adapt to the changes will get the best deal for themselves and for their patients.

1 Foster A. Developing the role of FHSAs. Primary Health Care Managemen $1991 ; 1(7): 2-3$.

2 NHS Management Executive. FHSAs-today's and tomorrow's priorities. London: Department of Health, 1991.

(Accepted 16 May 1991)

\section{THE MEMOIR CLUB}

My star patient was a small boy, not too bright, who had been taken by his mother (not too bright either) to the cinema to see Laurel and Hardy and who had laughed so immoderately that he had dislocated his jaw. The boy would not let me reduce the dislocation without an anaesthetic and because he had an anaesthetic the rule was that he had to be admitted overnight. He was discharged the next day with a stern admonition not to open his mouth too wide for some time. All was well for a week or two, then he returned in exactly the same condition, having been to see another Laurel and Hardy film. Again he needed an anaesthetic and again he was admitted; this time Laurel and Hardy were put firmly on the proscribed list. I did not see him again personally but I understand that over the next three or four years he was admitted regularly with the same condition, every time because he had been to the pictures.

Not all the patients I saw shared my enthusiasm for surgery and many of them said firmly: "There's tae be nae cuttin, Doctor." When "cuttin" was the only possible treatment $I$ had to argue, often for a long time, to get them to agree to it. On the other hand, some displayed a remarkable stoicism. I remember a small boy with badly burned buttocks. "Geordie Dolan pet me i' the fire," he explained. "When I get oot," he added meditatively, "Ah'll batter 'im!"

From Not a Proper Doctor by David Sinclair. Published under the BMF's Memoir Club imprint. ISBN 0727902792 . Price: Inland £14.95; abroad £18.50. BMA members: Inland $£ 13.95$; abroad $£ 17.50$. 